\title{
Parto prematuro y factores de riesgo asociados en el Hospital Universitario Metropolitano
}

\author{
Jaime Orozco Bayuelo*; Guillermo Acosta Osio MD**; Antonio Rojano Tejeda; Gustavo Rhenals D'Andreis***
}

RESUMEN: La finalidad del presente estudio fue determinar la incidencia de parto prematuro y factores de riesgo asociados en pacientes atendidas en el Hospital Universitario Metropolitano de Barranquilla, Colombia, durante 1993 y 1994. Se presentan resultados de 1.744 embarazos, de los que 140 fueron pretérmino para una incidencia de $8,48 \%$.

La edad materna menor de 17 años $\mathrm{Rr}: 1.4$ y mayores de $31 \mathrm{Rr}: 1.5$, el 54\% sólo había cursado primaria Rr: 1.9, eran nulíparas 45,7\% laboraban fuera del hogar 33,6\%; control prenatal inadecuado $(\mathbf{7 2 , 1 5 \%}$ ) hábito de fumar y DPPNI Rr: 5.3, 68,6\% tuvieron edad gestacional entre 34 y 36,5 semanas.

La primera patología fue ruptura prematura de membranas $(22,4 \%)$, infección urinaria $(\mathbf{1 2 , 3 \% )}$ fue la segunda, preeclampsia $(\mathbf{1 0 , 5 5 \%})$, anemia $(\mathbf{4 5 , 7 \% )}$. La predicción y prevención de los factores asociados es imperativo para reducir la prematurez.

PALABRAS CLAVES: Parto prematuro, nulíparas, control prenatal inadecuado, ruptura prematura de membranas, anemia.

SUMMARY: The purpose of this study was to determine the incidence of premature delivary and associated risk factors in patients which attended the Metropolitana University Hospital in Barranquilla, Colombia during the years 1993 and 1994.

The maternity age less than 17 years of age $\mathrm{Rr}: 1.4$ and older tham $31 \mathrm{Rr}: 1.5$, a $54 \%$, had only been them elementary school, $\mathrm{Rr}$ : 1.9 , had never had babies before $45.7 \%$ worked outside the home $33.6 \%$, inadecuate prenatal control (72.15\%) smoking habit and DPPNI Rr: $5.3,68.6 \%$ had a pregnancy stage among 34 and 36.5 weeks.

The first pathology was premature membrane rupture $(22.4 \%)$, urinary infection $(12.3 \%)$, was the second pre-eclamsy $(10.55 \%)$, anemia $(45.7 \%)$. The prediction and prevention of the associated factors is imperative to reduce the prematurity.

KEY WORDS: Premature delivery, inadecuate prenatal care, rupture premature membrane, anemie.

\section{Introducción}

El parto prematuro se define como el que ocurre después de las 20 y antes de las 37 semanas con un producto cuyo peso al nacer es mayor de 500 pero menor de 2.500 gramos (1-4). Se han relacionado diversos factores de riesgo entre ellos:

Edad materna $(3,5,8)$ raza, nivel socioeconómico (8), estado civil (9), tabaquismo (3, 8, 11-12), adición a la cocaína (3, 8-9), desnutrición materna (13-14), actividad física pesada (15-16), falta de control prenatal (17), antecedentes de parto prematuro $(1-3,5,8-9,18$, incompetencia itsmicocervical (19) anemia (19-22), infección materna (23-26), ruptura prematura de membranas (27-28).

El diagnóstico de la amenaza de parto prematuro se basa en edad del embarazo, características de las contrac-

\footnotetext{
* Ginecólogo Obstetra. Hospital Universitario Metropolitano.

** Director Departamento de Ginecología y Obstetricia. Hospital Universitario Metropolitano.

Coordinadores de Postgrado Ginecología y Obstetricia. Hospital Universitario Metropolitano.
}

ciones y estado del cuello uterino $(1-3,5-6,18)$, frecuentemente se habla de signos como presión pélvica, cólicos, sangrado vaginal, dolor en los muslos $(18,29-30)$; como también predictores bioquímicos como fibronectina fetal en secreción cérvico-vaginal (31-32). También es de utilidad el ultrasonido para visualizar acortamiento del cérvix, adelgazamiento del segmento uterino o protrusión de las membranas en el canal cervical (33-35).

El manejo dependerá si se trata de amenaza o trabajo de parto prematuro. Aunque lo ideal es el conocimiento de los factores asociados a la prematurez para así poder aplicar políticas de tipo preventivo tales como el mejorar condiciones de vida y escolaridad de las pacientes, al igual que ampliar la cobertura de atención prenatal.

El objetivo del presente trabajo es identificar los factores de riesgo mencionados y establecer su asociación al parto prematuro en nuestro medio.

Material y métodos

Se trata de un estudio descriptivo, epidemiológico observacional de tipo prospectivo, en el que se incluye- 
ron todas las pacientes con edad gestacional mayor de 20 y menor de 37 semanas, con un producto cuyo peso sea mayor de 500 pero menor de 2.500 gramos, que tuvieron parto vaginal o les fue practicada cesárea en el Hospital Universitario Metropolitano de Barranquilla, Colombia; entre enero 1o. de 1993 y diciembre 31 de 1994. Para calcular la edad gestacional se utilizó la fecha de la última menstruación confiable, y en las que no es posible, se recurrió a ayudas ecográficas; y en todos los neonatos se practicó evaluación por Capurro confirmatoria.

Todas las pacientes fueron entrevistadas por el investigador, llenando un protocolo previamente diseñado (el cual se anexa) para cumplir con los objetivos trazados. Los neonatos fueron evaluados por residentes o docentes de pediatría.

Se excluyeron de este estudio, los recién nacidos con peso menor de 2.500 gramos, cuya edad gestacional por amenorrea, es igual o mayor de 37 semanas; y los que teniendo menos de 37 semanas, pesan 2.500 gramos o más.

Se hizo análisis de riesgo, correlación de Pearson y se aplicó el test de Fisher's.

\section{Resultados y discusión}

Durante los 2 años del estudio, fueron admitidas al Hospital Universitario Metropolitano 1.744 pacientes para atención del parto; de las cuales, 29 cursaron con embarazo gemelar obteniéndose 1.733 recién nacidos.

Se seleccionaron 140 pacientes que tuvieron parto prematuro, con un resultado de 150 recién nacidos, por lo que la incidencia fue de $8,48 \%$ (Tabla 1 y Figura 1); siendo similar a lo establecido para América Latina por Schwarcz y colaboradores (6), inferior a lo hallado por Cifuentes (3) en Cali: $12.9 \%$ y Botero y Jubiz (5) en Medellín: $12,3 \%$ pero un poco más alto que el informado por Monterrosa (7) en Cartagena.

Tabla 1

\section{INCIDENCIA DE PARTO PREMATURO SEGUN AÑO}

\begin{tabular}{|l|rc|rr|rr|}
\hline \multirow{2}{*}{ Año } & \multicolumn{2}{|c|}{1993} & \multicolumn{2}{c|}{1994} & \multicolumn{2}{c|}{ Total } \\
Nacimientos & No. & \multicolumn{1}{c|}{$\%$} & No. & \multicolumn{1}{c|}{$\%$} & No. & \multicolumn{1}{c|}{$\%$} \\
\hline A término & 771 & $90.53 \%$ & 852 & 92,51 & 1.623 & 91,50 \\
Prematuros & 81 & $9.47 \%$ & 69 & 7,49 & 150 & 8.48 \\
\hline Total & 852 & $100 \%$ & 921 & $100 \%$ & 1.773 & $100 \%$ \\
\hline
\end{tabular}

Fuente: Toda la información que se presenta en este trabajo procede de Historias Clínicas de pacientes atendidas en el Hospital Universitario Metropolitano.

La edad materna en la que se encontró el mayor número de partos prematuros fue entre 18 y 30 años, siendo éste el grupo etario con más frecuencia en la población general estudiada, como muestra la tabla 2 y figura 2; esto contrasta con lo informado en otros estudios que señalan las edades extremas $(3,8)$. Aquí se halló un riesgo relativo de 1,4 para las madres menores o de 17 años y de 1,5 para las mayores o de 31 años.
Figura 1

\section{INCIDENCIA DE PARTO PREMATURO SEGUN AÑO}

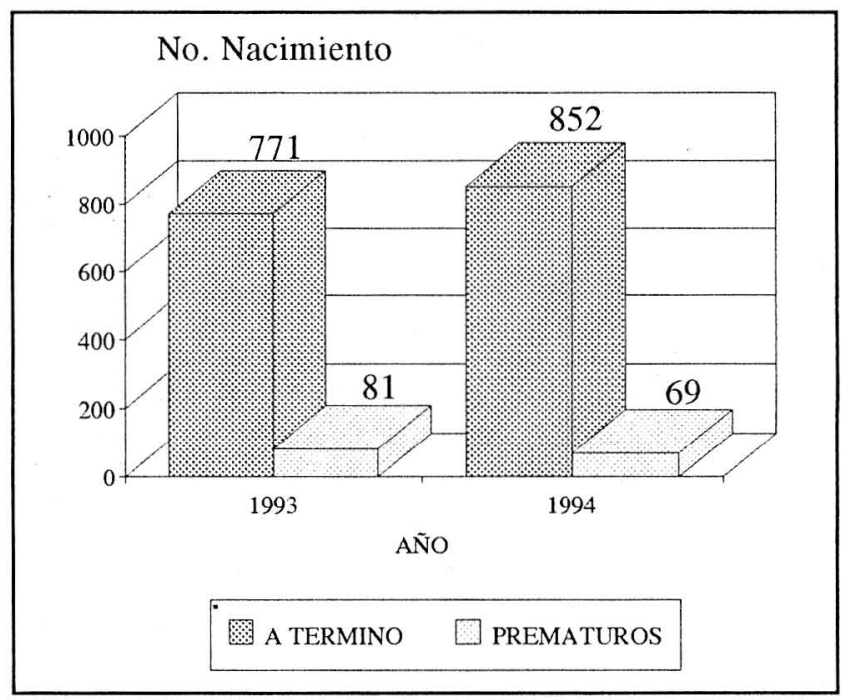

Tabla 2

INCIDENCIA DE PARTO PREMATURO SEGUN EDAD MATERNA

\begin{tabular}{|l|rr|rr|r|}
\hline & \multicolumn{2}{|c|}{ Prematuros } & \multicolumn{2}{|c|}{ A término } & \\
& No. & \multicolumn{1}{c|}{$\%$} & No. & \multicolumn{1}{c|}{$\%$} & Total \\
\hline < 17 Años & 10 & $0,57 \%$ & 95 & 5,45 & 102 \\
$18-30$ Años & 102 & 5,85 & 1200 & 68,80 & 1.302 \\
$>-31$ Años & 28 & 1,62 & 309 & 17,71 & 337 \\
\hline Total & 140 & 8,04 & 1604 & $91,96 \%$ & 1.744 \\
\hline
\end{tabular}

\section{Figura 2}

\section{INCIDENCIA DE PARTO PREMATURO SEGUN EDAD MATERNA}

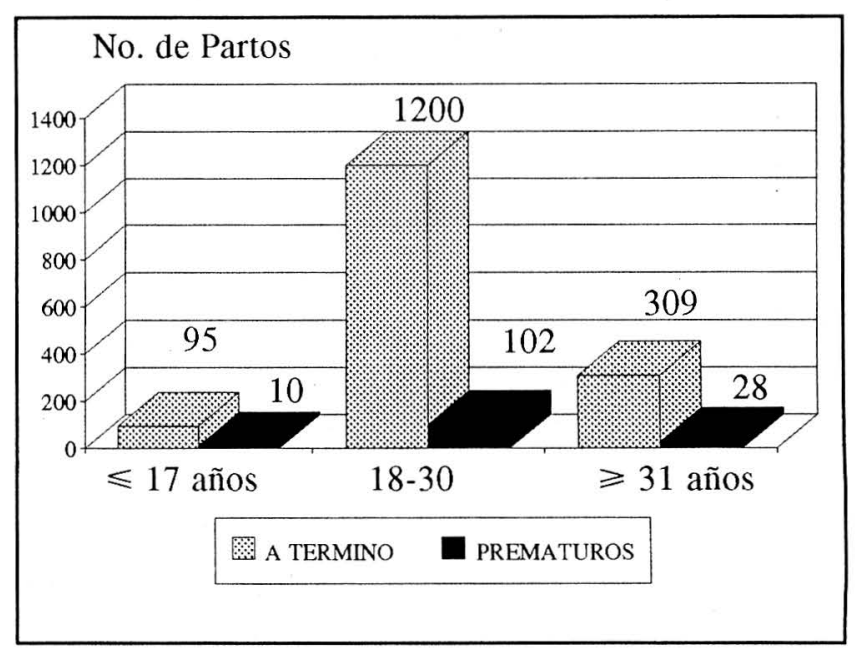


De las pacientes estudiadas el $85,7 \%$ (120) residía en área urbana y sólo un $14,3 \%$ (20) en área rural; con un riesgo relativo de 1,3 cuando la residencia estaba en área rural. (Tabla 3 ).

\section{Tabla 3}

PARTO PREMATURO EN RELACION CON LUGAR DE RESIDENCIA

\begin{tabular}{|l|cc|cc|c|}
\hline Parto prematuro & \multicolumn{2}{|c|}{ Si } & \multicolumn{2}{|c|}{ No } & \multirow{2}{*}{ No. \% } \\
Lugar de Residencia & No. & $\%$ & & \\
\hline Urbano & 120 & 6.9 & 1376 & 78.89 & 1496 \\
Rural & 20 & 1.14 & 228 & 13,07 & 248 \\
\hline Total & 140 & 8.04 & 1604 & 91.96 & 1744 \\
\hline
\end{tabular}

Hubo una inestabilidad conyugal del $20 \%$ (18 pacientes) en riesgo relativo de 1.3; hay autores que afirman que ésta acarrea inseguridad y quieren esconder el embarazo de la sociedad, acompañado también de dieta pobre y un inadecuado control prenatal.

Se suele utilizar la escolaridad como una aproximación del nivel socio económico (18), aquí se halló que 74 de las pacientes con parto prematuro $(54,3 \%)$ habían cursado algún grado de educación primaria, mientras que el $40,7 \%$ (59) habían cursado parcial o completamente la secundaria y sólo 7 pacientes habían realizado o estaban realizando estudios universitarios (Fig. 3). No hubo analfabetismo, pero la mayoría de los pacientes tenía bajo nivel educativo. El riesgo relativo para la primaria fue de 1.9 .

Estaban dedicadas a labores propias del hogar, el $66.4 \%$ (93) mientras que $47(33.6 \%)$ lo hacían fuera de éste (Fig. 4), especialmente servicio doméstico y otras actividades que requerían de esfuerzo físico. Se afirma que el cargar niños u objetos pesados y realizar esfuerzo, puede inducir contracciones uterinas $(8,15-16)$.

Figura 3

ESCOLARIDAD

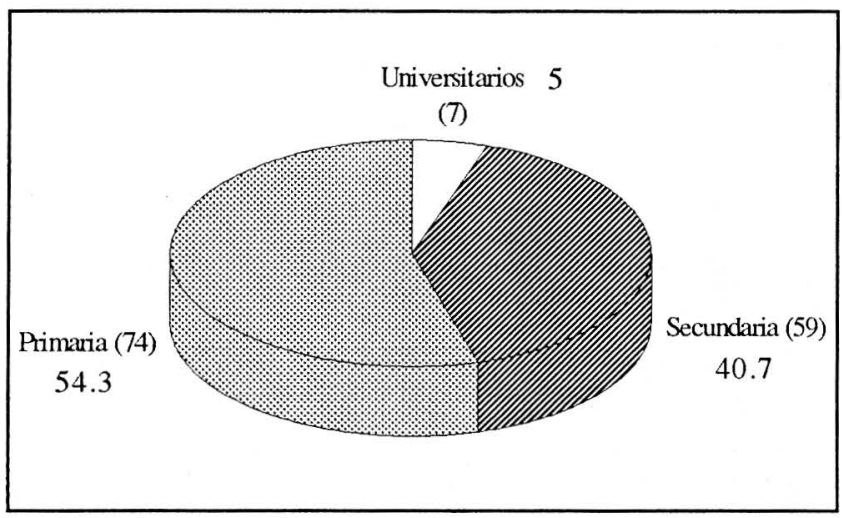

Figura 4

PARTO PREMATURO Y ACTIVIDAD MATERNA

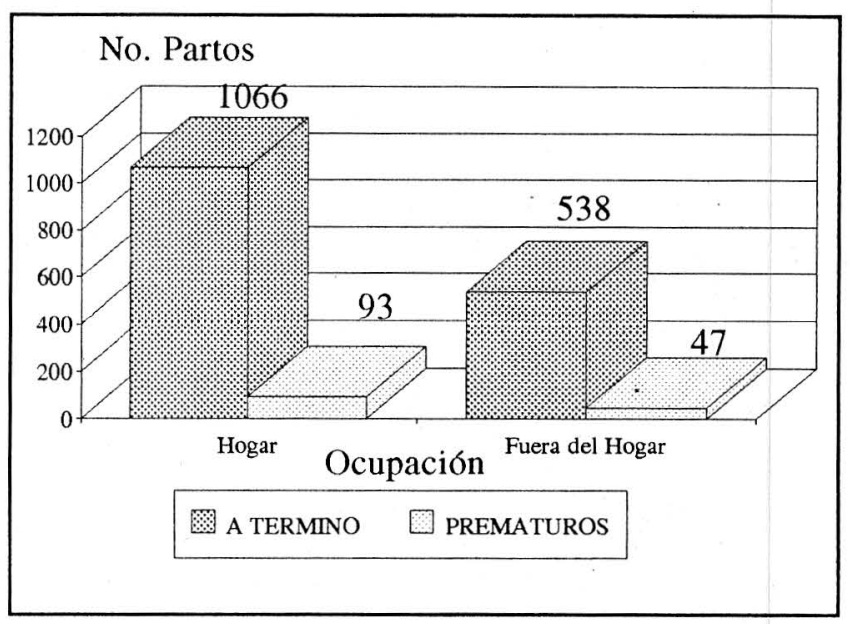

Se estudió la estabilidad laboral del cónyuge como índice de estabilidad económica, mostrando que sólo el $28,6 \%$ (40) eran inestables con un riesgo relativo de 1.2.

Varios autores entre ellos Fernando Arias mencionan que las nulíparas son tres veces más propensas a tener parto prematuro, como también la gran multiparidad (4 o más embarazos). Aquí se estableció que 64 pacientes $(45,7 \%)$ eran nulíparas y $31(22,1 \%)$ pertenecían al 20 . grupo de riesgo (Tabla 4 y Figura 5), hubo un riesgo relativo de 1,8 para nuliparidad y de 1,4 para gran multiparidad.

Tabla 4

PARTO PREMATURO Y PARIDAD

\begin{tabular}{|l|cc|cc|c|}
\hline Prematurez & \multicolumn{2}{|c|}{ Si } & \multicolumn{2}{|c|}{ No } & \\
& No. & \% & No. & \% & Total \\
\hline Paridad & & & & & \\
Nulíparas & 64 & 3.67 & 733 & 42,03 & 797 \\
Multíparas & 76 & 4.36 & 871 & 49.94 & 947 \\
\hline Total & 140 & 8.03 & 1604 & 91.97 & 1744 \\
\hline
\end{tabular}

Treinta y cuatro pacientes tenían antecedentes de aborto $(24,3 \%)$ con un riesgo relativo de 1,3 y solamente el $12.5 \%$ (17 pacientes), tenían antecedentes de partos prematuros.

Cuando se realiza un control prenatal adecuado (4 ó más consultas), se disminuye el riesgo de parto prematuro, sólo 39 pacientes (27.85\%) lo hicieron: en el 53,57\% (75 pacientes) fue inadecuado y el $18,58 \%$ (26) no lo hizo, éstas últimas provenían de áreas rurales o sólo habían cursado educación primaria como lo muestra la tabla 5 . 
Figura 5

PARTO PREMATURO Y PARIDAD

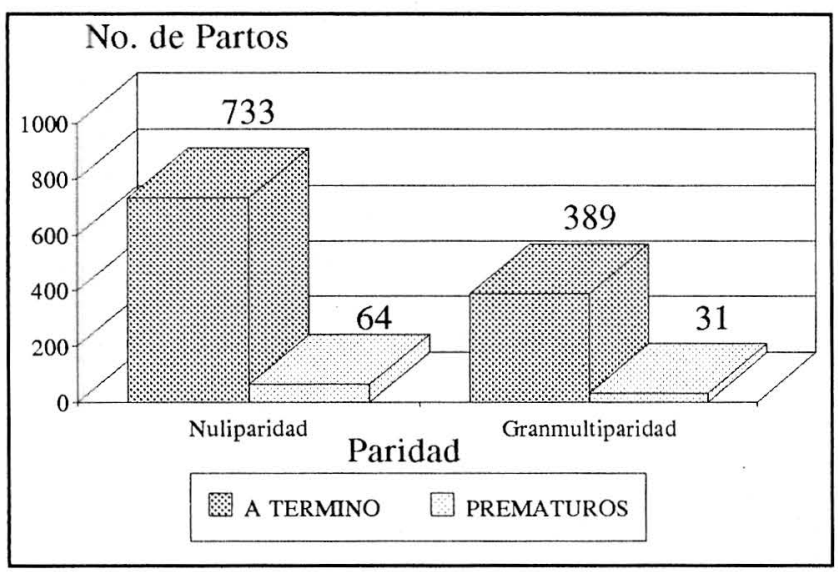

Tabla 5

CONTROL PRENATAL Y EDUCACION

\begin{tabular}{|l|c|c|c|}
\hline $\begin{array}{l}\text { Educación } \\
\text { Control Prenatal }\end{array}$ & 1ria. & 2daria. & U. \\
\hline Adecuado & 18 & 20 & 1 \\
Inadecuado & 38 & 31 & 6 \\
No hizo & 18 & 8 & \\
Total & 74 & 59 & 7 \\
\hline
\end{tabular}

En las madres estudiadas se encontró que 19 eran fumadoras lo que equivale a un 13.6\% (Figura 6). Hallándose asociación entre hábito de fumar y D.P.P.N.I. en 5 de los 11 casos que se presentaron, lo que mostró un riesgo relativo de 5.3.

La vía del parto para los 150 recién nacidos fue por cesárea en 79 casos (52.71) y vaginal en 71 (47.3\%) (Fig. 7) observándose que la gran mayoría de las presentaciones pélvicas se resolvieron por cesárea y que sólo 4 de éstas (2.6\%) nacieron por vía vaginal (Tabla 6).

Figura 6

\section{PARTO PREMATURO Y HABITO DE FUMAR}

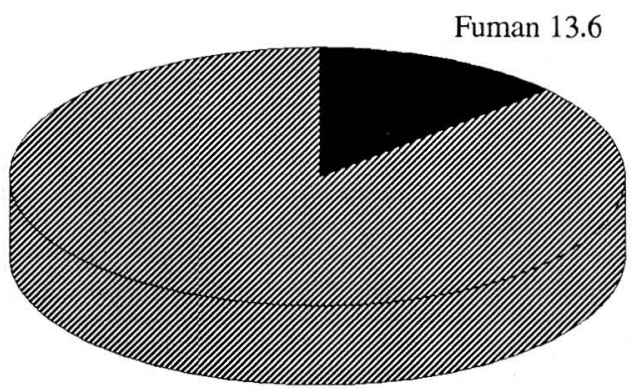

No fuman 86.4
Tabla 6

VIA DEL PARTO Y PRESENTACION FETAL

\begin{tabular}{|l|cc|cc|cc|c|}
\hline $\begin{array}{l}\text { Presentación } \\
\text { Vía del Parto }\end{array}$ & \multicolumn{2}{|c|}{ Cef. } & \multicolumn{2}{c|}{ Pelv. } & \multicolumn{2}{|c|}{ Transv. } & \\
\hline Vaginal & 67 & 44.7 & 4 & 2.6 & - & - & 71 \\
Cesárea & 55 & 36.7 & 21 & 14.0 & 3 & $2 \%$ & 79 \\
\hline Total & 122 & 81.4 & 25 & $16.6 \%$ & 3 & $2 \%$ & 150 \\
\hline
\end{tabular}

En cuanto a la edad gestacional al nacer, se encontró que el 68.6\% (96 pacientes) tenían entre 34 y 36.9 semanas; siendo el grupo menos numeroso el de una edad gestacional de 30 semanas o menos (ver figura 8), lo cual coincide con varios informes publicados $(3,5,7)$. Ver figura 8 .

La casi totalidad de los recién nacidos con 34-36,9 semanas de gestación al nacer, pesaban entre 1.501 y 2.490 gramos (Tabla 7) y el $47 \%$ de los que tenían 30 semanas o menos su peso estuvo por debajo de 1.000 gramos, la correlación observada fue, $\mathrm{Rr}$ : 0,91 es decir, alta correlación.

Figura 7

\section{VIA DEL PARTO}

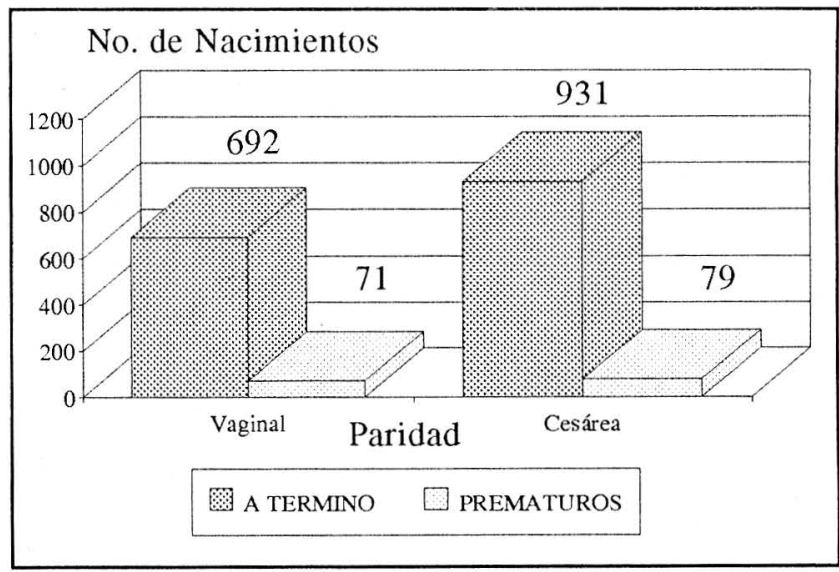

Figura 8

EDAD GESTACIONAL AL NACER

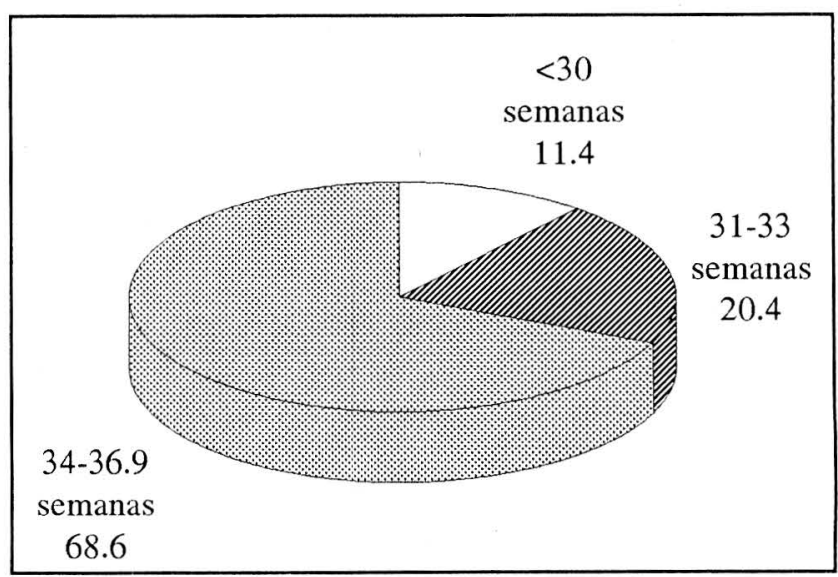


Tabla 7

EDAD GESTACIONAL Y PESO AL NACER

\begin{tabular}{|l|c|c|c|c|}
\hline $\begin{array}{l}\text { Peso al nacer } \\
\begin{array}{l}\text { Edad } \\
\text { gestacional }\end{array}\end{array}$ & $\begin{array}{c}<\mathbf{1 . 0 0 0} \\
\text { grs. }\end{array}$ & $\begin{array}{c}\mathbf{1 . 0 0 1 - 1 . 5 0 0} \\
\text { grs. }\end{array}$ & $\begin{array}{c}\mathbf{1 . 5 0 1 - 2 . 4 9 9} \\
\text { grs. }\end{array}$ & Total \\
\hline$<30$ Sem. & 8 & 5 & 4 & 17 \\
31-33 Sem. & 1 & 10 & 16 & 27 \\
34-36 Sem. & - & 1 & 105 & 106 \\
\hline Total & 9 & 16 & 125 & 150 \\
\hline
\end{tabular}

No se halló diferencia notable en cuanto al sexo del recién nacido, 77 femeninos $(51,4 \%)$ y 73 masculinos (48,6\%). (Fig. 9).

En los 150 recién nacidos hubo 10 óbitos, quedándonos 140 nacidos vivos, de ellos al primer minuto el $78,6 \%$ tuvieron un Apgar de 7 ó mayor; siendo en los de término $84,3 \%$. Al quinto minuto solamente dos de los prematuros tenían Apgar inferior a 7.

Figura 9

\section{SEXO DEL RECIEN NACIDO}

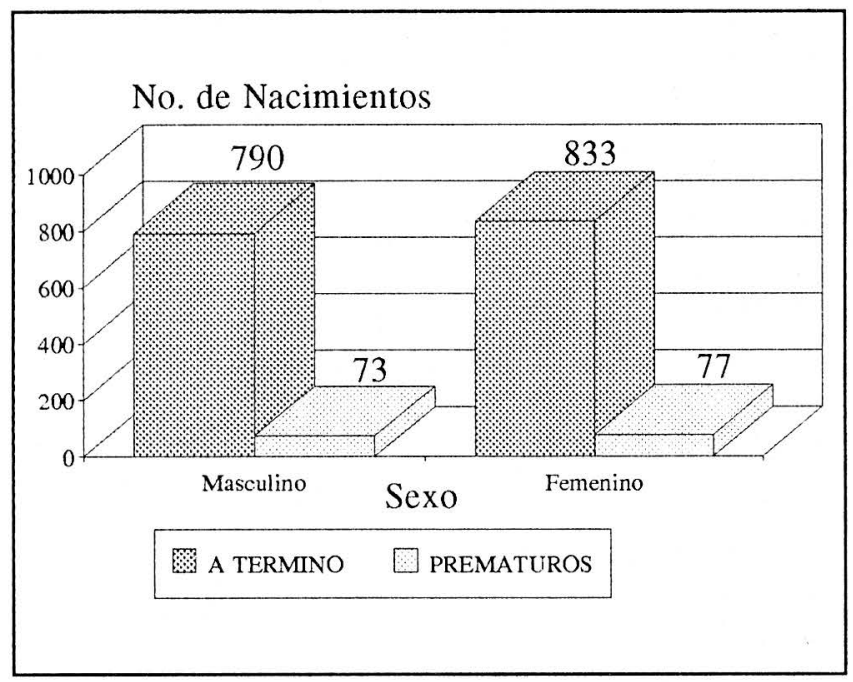

Al comparar con la edad gestacional encontramos que el mayor número de óbitos fetales estaba entre 31 y 34 semanas (Tabla 8) y el menor número en el grupo de 3436,9 semanas.

La patología más frecuentemente hallada asociada al parto prematuro fue Ruptura prematura de membrana en $22,5 \%$, seguido de las infecciones urinarias $12,3 \%$ (Tabla 9 ).

Hay algunas patologías que obligan a un manejo obstétrico tales como preeclampsia, que alcanzó $10,55 \%$, DPPNI y placenta previa con 5 y $4,5 \%$ respectivamente. Está demostrado que estas dos últimas ocurren en un gran porcentaje antes de las 37 semanas.
Tabla 8

RELACION ENTRE EDAD GESTACIONAL Y OBITO FETAL

\begin{tabular}{|l|c|c|c|}
\hline $\begin{array}{l}\text { Parto prematuro } \\
\text { Edad gestacional }\end{array}$ & Vivos & Obitos & Total \\
\hline$<30$ semanas & 13 & 3 & 16 \\
$31-33$ sem. & 23 & 5 & 28 \\
$34-36,9$ sem. & 94 & 2 & 96 \\
\hline Total & 140 & 10 & 150 \\
\hline
\end{tabular}

Tabla 9

PATOLOGIAS ASOCIADAS AL PARTO PREMATURO Y EDAD GESTACIONAL

\begin{tabular}{|c|c|c|c|c|c|}
\hline $\begin{array}{l}\text { Edad gestacional } \\
\text { Patología asociada }\end{array}$ & $\begin{array}{l}<30 \\
\text { Sem. }\end{array}$ & $\begin{array}{l}31-33 \\
\text { sem. }\end{array}$ & $\begin{array}{l}34-36,9 \\
\text { Sem. }\end{array}$ & $\begin{array}{r}\text { To } \\
\text { No. }\end{array}$ & $\%$ \\
\hline \multicolumn{6}{|l|}{ Ruptura Prematura } \\
\hline Membrana & 4 & 11 & 34 & 49 & $22.4 \%$ \\
\hline Infección de vías & & & & & \\
\hline urinarias & 2 & 6 & 19 & 27 & $12,3 \%$ \\
\hline Preeclampsia & 1 & 9 & 13 & 23 & $10,55 \%$ \\
\hline Vaginitis & 1 & 5 & 13 & 19 & $8,7 \%$ \\
\hline D.P.P.N.I.* & 2 & 4 & 5 & 11 & $5,0 \%$ \\
\hline Placenta previa & 2 & 2 & 6 & 10 & $4,5 \%$ \\
\hline Embarazo gemelar & 1 & 1 & 8 & 10 & $4,5 \%$ \\
\hline Corioamnionitis & 1 & 1 & 3 & 5 & $2,29 \%$ \\
\hline Anemia & 9 & 11 & 44 & 64 & $29,3 \%$ \\
\hline
\end{tabular}

* Desprendimiento prematuro de placenta normoimplantada.

Estudios recientes nos muestran la importancia de la flora vaginal materna en el parto prematuro (25-26), aquí la vaginitis se asoció en el $8,7 \%$ de las pacientes.

Desde hace mucho tiempo se ha mencionado la gestación múltiple como factor de riesgo para el parto prematuro $(3,5,8-9)$, la asociación hallada fue de $4,5 \%$ ya que hubo embarazo gemelar en 10 pacientes, con un riesgo relativo de 4,5 .

La corioamnionitis, que en todos los casos estuvo acompañada de R.P.M. se presentó en 5 casos (2,29\%), se ha llegado a afirmar que en algunos casos puede proceder a la ruptura prematura de membranas (27-28).

Vale la pena mencionar que en 64 de las 140 pacientes estudiadas $(45,7 \%)$ se encontró anemia (hemoglobina por debajo de 11 gramos) (3); hay varios estudios que asocian la culminación insatisfactoria del embarazo a la anemia materna $(9,20-22)$.

Los resultados obtenidos en cuanto a patologías asociadas, son similares a las publicadas por Arias (18) y por Garbaciak (9) y a los hallados para otras regiones del país, $(3,5)$ especialmente en la Costa Atlántica (7). 


\section{Conclusiones}

La incidencia de Parto Prematuro hallado fue: $8.48 \%$.

Para las madres de 17 años o menos se encontró un riesgo relativo de 1.4 de parto prematuro, mientras que para las de 31 años o más el riesgo relativo fue de 1.5 veces.

El residir en área rural: tuvo un riesgo relativo de 1.3 veces.

La inestabilidad conyugal tuvo un riesgo relativo de 1.3 veces.

El $54,3 \%$ de las pacientes sólo había cursado parcial o totalmente la primaria: con riesgo de 1.9 veces.

El laborar fuera del hogar se encontró en $33.6 \%$.

$\mathrm{La}$ inestabilidad laboral del cónyuge (Económica) tuvo un riesgo relativo de 1,2 .

El $45.7 \%$ lo eran nulíparas, se halló un riesgo relativo de 1,8 veces, mientras que en las grandes multíparas hubo $22.1 \%$.

Hubo aborto previo en el $24,3 \%$ de pacientes con un riesgo relativo de 1,5 veces.
Hubo parto prematuro previo en $12,5 \%$ de pacientes.

El control prenatal fue inadecuado en el $72,15 \%$ de las pacientes.

El riesgo relativo entre hábito de fumar y D.P.P.N.I. fue de 5,3 veces.

No hubo diferencia significativa en cuanto a vía del parto; el $84 \%$ de los podálicos nacieron por cesárea y el $54,4 \%$ de los cefálicos por vía vaginal.

La edad gestacional al nacer del $68,6 \%$ fue entre 34 y 36,9 semanas.

El Apgar de los recién nacidos fue bueno en el 78,6\% de los casos.

La primera patología asociada a Parto Prematuro fue Ruptura Prematura de Membranas en 22,4\%.

La segunda causa fue la Infección de Vías Urinarias en $12,3 \%$.

Embarazo Múltiple se encontró en el $4,5 \%$ de las pacientes.

La vaginitis estuvo asociada en el $8,7 \%$ de los casos. Se halló Anemia en el 45,7\% de las pacientes.

\section{BIBLIOGRAFIA}

1. Acosta Ossio G. Obstetricia y Ginecología. Manual de Normas. 2a. Edición. Editorial Espriellabé. Barranquilla. 1992.

2. Iffi L., Kaminetzky H. Obstetricia y Perinatología. 2a. Reimpresión. Editorial Panamericana. Bs. Aires. 1990.

3. Cifuentes R. Obstetricia de Alto Riesgo. 4a. Edición. Editorial Aspromédica. Cali. 1994.

4. Altirriba E. y otros. Prematuridad (I). Clin. Invest. Ginecol. Obstet. 1991; 18(8)

5. Botero J., Jubiz-A. y Henao-G. Obstetricia y Ginecología. 4a. Edición. Editorial Carvajal. Medellín, 1992.

6. Schwarcz R., Duverges C., Díaz-A.G. y Fescina R. Obstetricia. 4a. Edición- Reimpresión. Editorial el Ateneo, Buenos Aires. 1989.

7. Monterrosa A. Incidencia de Parto Pretérmino y factores de Riesgo. Rev. Col. Obstet. Ginecol. 1991; 42(3).

8. Main D. Epidemiología del Parto Pretérmino. Clínicas Obst. Ginecol. Editorial Interamericana 1988; 3.

9. Garbaciak J. Prevención de la Prematurez: ¿Quién est en Peligro? Clínicas de Perinatología-Editorial Interamericana. 1992; 2.

10. Papiernik E. Predicción del Parto Pretérmino. Rev. Galémica 1987; $1(5)$.

11. Williams M., Mittendorf $\mathrm{R}$ et al. Cigarretes, Coffe, and Preterm Premature Rupture of the Membrans. Obstetrical and Gynecological Survey 1993; 48(2): 69-71.

12. Narahara H., Johnston JM. Smoking and preterm labor. Effect of cigarrette smoke extract on the Secretion of platelet activating factor acetylhydrolase by human decidual macrophages. Am. J. Obstet. Gynecol. 1993; 69(5): 134-136.

13. Kristal A. Nutrición Materna y duración de la gestación. Clínica Obstétrica y Ginecológica. Editorial Interamericana. 1984; 3.

14. Wildschut Hij. Determinants of preterm birth in Curacao, Netherlands Antilles. Int. J. Ginecol. Obstet. 1991; 36: 23-27.

15. Papiernik E. Propuestas para una política programada de prevención del parto pretérmino. Clínicas obstétricas y Ginecológicas. Editorial Interamericana. $1984 ; 3$.

16. Valencia L.M. Factores de Riesgo del Parto Prematuro en gestantes trabajadoras. Rev. Colombiana de Obstetricia y Ginecología. 1992; 43(2).

17. Scholl TO et al. Prenatal care adequancy and the outcome of adolescent pregnancy: Effects on weight gain, preterm delivery and birth weight. Obstet. Gynecol. 1987; 69(3): 312-316.

18. Arias F. Practical Guide to High Risk Pregnancy and Delivery. 2a. Edición. Editorial Mosby Year Book. St. Louis. 1993.
19. Parisi V. Incompetencia cervical y trabajo de parto pretérmino. Clínicas Obstétricas y Ginecológicas Editorial Interamericana. 1988; 3.

20. Iams J., Jonhson F. y Creasy R. Prevención del parto pretérmino. Clínicas Obstétricas y Ginecológicas. Editorial Interamericana 1988; 3.

21. Klebanoff MA., Shiono PH et al. Anemia and espontaneus preterm birth. Am. J. Obstet. Gynecol. 1991; 164: 59-63.

22. Lieberman E., Ryan $\mathrm{KJ}$ et al. Association of maternal hematocrit with premature labor. Am. J. Obstet. Gynecol. 1988; 159.

23. Romero R. y Mazor M. Infección y trabajo de parto pretérmino. Clínicas Obstétricas y Ginecológicas. Editorial Interamericana. $1988 ; 3$.

24. Cassey ML., MC Donal P. Procesos biomoleculares en el inicio del parto: activación decidual. Clínicas Obstétricas y Ginecológicas. $1988 ; 3$.

25. MC Gregor JA., French JI et al. Anteneonatal microbiologic and maternal risk factors associated with Prematurity. Am. J. Obstet. Gynecol. 1990; 163: 1465-1475.

26. Krohn $M$ et al. Vaginal bacteriodes species are associated with an increased rate of preterm delivery among women in preterm labor. Obstetrical and Gynecological Survey 1991; 47(1): 14-15.

27. Romero R., Ghidini A. Invasión microbiana de la cavidad amniótica en la ruptura de membranas. Clínicas Obstétricas y Ginecológicas. 1991; 4 (737-745).

28. Cambell BA., Newman RB. Uterine activity after preterm premature rupture of the membranes. Am. J. Obstet. Gynecol. 1991; 165: 422425.

29. Iams J., Stilson R et al. Symptoms that precede preterm labor and preterm premature rupture of the membranes. Am. J. Obstet. Gynecol. 1990; 162: 486-490.

30. Katz M., Goodyear K., Creasy R. Early signs and symptons of preterm labor. Am. J. Obstet. Gynecol. 1990; 162: 1250-1253.

31. Lockwood CJ., Wein R., Lapinsky R et al. The presence of cervical and vaginal fetal fibronectin predicts preterm delivery in an inner-city obstetric population. Am. J. Obstet. Gynecol. 1993; 169 (94) 798804. J. Obstet. Gynecol. 1994; 170(1): 20-25.

32. Nageotte MP., Casal D., Senyei AE. Fetal fibronectin in patients at increased risk for premature birth. Am. J. Obstet. Gynecol. 1994; 170(1): 20-25.

33. Okitsu O., Mimura T., Nakayama T. Early prediction of preterm delivery by transvaginal ultrasonography. Obstet. Gynecol. Survey. 1993; 48(8): 519-521. 
34. Andersen HF., Nugent $C$ et al. Prediction of risk for preterm delivery by ultrasonographic measurement of cervical length. Am. J. Obstet. Gynecol. 1990; 63: 859.

35. Murakawa H., Utumi T., Hase Gawa I. and Tanaka K. Evaluation of threatened preterm deliyery iv transvaginal ultrasonographic. Obstet. Gynecol. 1993; 82(5): 829-832.

36. Leonardi M. y Hankins G. Novedades en el campo de los Tocolíticos. Clínicas de Perinatología. 1992; (2): 367-383.

37. Higby K., Xenakis E and Paverstein C. Do tocolytic agents stop preterm labor? A critical and comprehensive review of efficacy and safety. Am. J. Obstet. Gynecol. 1993; 168: 1247-1259.

38. Morales W. and Madhay H. Efficacy and safety of indmetacin compared with magnesium Sulfate in the management of preterm labor. A randomized study. Am. J. Obstet. Gynecol. 1993; 169: 97-102.

39. Major CA., Lewis DF., Harding JA et al. Tocolysis with Indometacin in creases the incidence of Necrotizing Enterocolitis in the low birth weight neonate. Am. J. Obstet. Gynecol. 1994; 170: 102-106.

40. Glock JL., Morales W. Efficacy and safety of Nifedipine versus Magnesium Sulfate in the management of preterm labor: A randomized study. Am. J. Obstet. Gynecol. 1993; 169(4): 960-964.
41. Madden CH., Owen J. and Hauth J. Magnesium tocolysis: Serum levels versus success. Am. J. Obstet. Gynecol. 1990; 162: 1177-1180.

42. Eschenbach DA., Nugent RP et al. A randomized placebo controled trial of Eritromicin for the treatment of Ureaplasma Urealyticum to prevent premature delivery Am. J. Gynecol. 1991; 164: 734-742.

43. MC Gregor JA., French JI., Seo K. Adjunctive Clindamicyn therapy for preterm labor. Result of a double blind, placebo controlled trial. Am. J. Obstet. Gynecol. 1991; 165: 867-875.

44. MC Gregur JA., French JI., Jones MS et al. Bacterial Vaginosis is associated with prematurity and vaginal fluid mucinase and salidace: Results of a controlled trial of topic Clindamycin Cream. Am. J. Obstet. Gynecol. 1994; 170: 1048-1060.

45. Morales WJ., Angel JL., O'Brien WF et al. A randomized study of antibiotic therapy in idiopatic preterm labor. Obstet. Gynecol. 1988; 72: 829-833.

46. Romero R., Sibai B., Caritis S., Paul R., Depp R et al. Antibiotic treatment of preterm labor with intac membranes. A multicenter randomized, double blinded, placebo controlled trial. Am. J. Obstet. Gynecol. 1993; 169: 764-774.

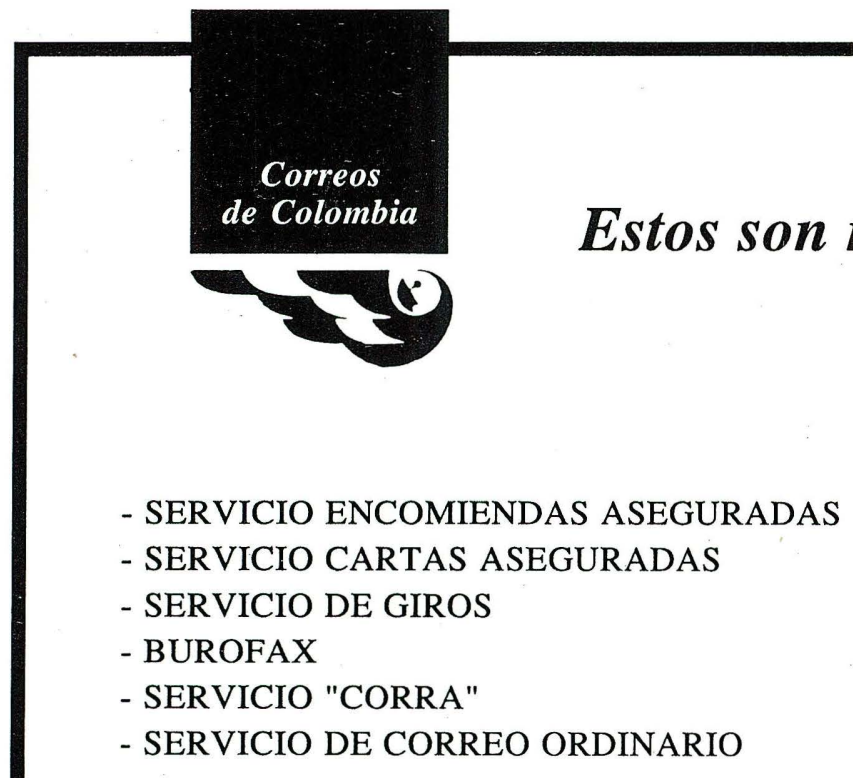

- SERVICIO DE CORREO CERTIFICADO

- SERVICIO DE CERTIFICADO ESPECIAL

- ENCOMIENDAS CONTRA REEMBOLSO

- SERVICIO TARIFA POSTAL REDUCIDA

- SERVICIO DE FILATELIA

- SERVICIO ELECTRONICO

- SERVICIO INTERNACIONAL APR/SAL

- SERVICIO RESPUESTA COMERCIAL

- SERVICIOS ESPECIALES

Teléfonos para quejas y reclamos: 3340304 - 3415536 - Santafé de Bogotá, D.C. - Colombia Cuente con nosotros hay que creer en los Correos de Colombia 